\title{
Local current mapping of electrochemically-exfoliated graphene oxide by conductive AFM
}

\section{$\operatorname{AUTHOR}(S)$ :}

Kubota, Wataru; Utsunomiya, Toru; Ichii, Takashi; Sugimura, Hiroyuki

\section{CITATION:}

Kubota, Wataru ... [et al]. Local current mapping of electrochemically-exfoliated graphene oxide by conductive AFM. Japanese Journal of Applied Physics 2020, 59(SN): SN1001.

\section{ISSUE DATE:}

2020-08-01

URL:

http://hdl.handle.net/2433/250520

\section{RIGHT:}

This is a peer-reviewed, un-copyedited version of an article accepted for publication/published in Japanese Journal of Applied Physics. IOP Publishing Ltd is not responsible for any errors or omissions in this version of the manuscript or any version derived from it. The Version of Record is available online at https://doi.org/10.35848/1347-4065/ab80df;; The full-text file will be made open to the public on 9 April 2021 in accordance with publisher's 'Terms and Conditions for Self-Archiving'.; この論文は出版社版でありません。引用の際には出版社版をご確認ご利用ください。; This is not the published version. Please cite only the published version. 


\title{
Local current mapping of electrochemically-exfoliated graphene oxide by conductive AFM
}

Wataru Kubota, Toru Utsunomiya, Takashi Ichii and Hiroyuki Sugimura

Department of Materials and Engineering, Kyoto Univ., Sakyo, Kyoto 606-8501, Japan

E-mail: utsunomiya.toru.5v@kyoto-u.ac.jp

\begin{abstract}
Electrochemical exfoliation of graphite is one of the promising method for the mass-production of graphene oxide (GO). The analysis of chemical condition, structure, and the electrical property of graphene-like materials, which is usually evaluated by X-ray photoelectron spectroscopy (XPS) or micro Raman spectroscopy ( $\mu \mathrm{RS}$ ), is important for the practical application. However, the information obtained by these instruments are spatially averaged. In this report, electrical property of mono-layer electrochemically-exfoliated GO (EGO) sheet was directly measured by conductive atomic force microscopy (C-AFM). Although there was little difference between EGO and GO synthesized by conventional chemical oxidation routes (CGO) in terms of chemical and structural characteristics, the electrical conductivity of EGO was much higher $(27000 \mathrm{~S}$ $\mathrm{m}^{-1}$ ) than that of CGO which is considered as the insulating material. These results proved the significance of C-AFM for unveiling the relationship between the structure and the electrical properties of graphene-like materials.
\end{abstract}




\section{Introduction}

Graphene is drawing much attention due to its outstanding properties of high electrical conductivity $^{1)}$, mechanical strength ${ }^{2}$, thermal conductivity ${ }^{3)}$, and so on. Many researchers have been making efforts not only to reveal its physical and chemical property for several application, for example, bio-sensor ${ }^{4)}$, transparent conductors $^{5)}$, supercapacitors $^{6}$, but also to produce it on a large scale cost-effectively. For the synthesis of graphene, there are various types of methods such as mechanical exfoliation, chemical vapor deposition $\left.(\mathrm{CVD})^{7}\right)$, epitaxial growth ${ }^{8)}$, and reduction of graphene oxide $\left.(\mathrm{GO})^{9}\right)$. In the case of CVD or epitaxial growth, high quality graphene can be synthesized. However, it is inevitable for the graphene synthesized by CVD process or epitaxial growth to transfer to the desired substrates ${ }^{10)}$ and it is not suitable for the mass-production of graphene. On the other hand, GO can be fabricated by graphite oxidation and, moreover, GO sheets can be dispersed in water or some organic solvents owing to epoxide, hydroxyl and carboxyl groups on GO sheets $^{11)}$. Hence GO can be directly deposited on the substrate by simple and inexpensive solution process and GO reduction is a promising method toward the mass-production of graphene commercially. However, the electrical property of reduced GO is inferior to that of graphene synthesized by other process because there are many defects on the GO sheets formed during the oxidation process, and these defects at the GO sheets can not be repaired easily by the reduction process, which deteriorates the performance of reduced GO.

Recently, new method to synthesize GO has been focused, that is, the exfoliation of graphite by anodization. When the positive voltage was applied to graphite in the electrolyte of some aqueous solution of sulfates ${ }^{12-15)}$, alkali halide ${ }^{16)}$ or ionic liquid ${ }^{17)}$, ions or water molecules were inserted into the graphite layers, and then graphite was exfoliated into multi or mono-layer GO sheets by the gas pressure produced by intercalated species decomposition. In this process, it takes less than 1 hour for the graphite exfoliation, so GO can be synthesized rapidly. Furthermore, there are fewer defects on electrochemicallyexfoliated GO (EGO) than that synthesized by chemically oxidized process, for example, Hummers method because graphite is moderately oxidized without strong oxidant in a short reaction time.

The electrical property of graphene like materials is very important for its practical application of electronics. It is often reported that oxygen functional groups or defects on the GO sheet degrade its electrical property by the chemical and structural analysis of the X-ray photoelectron spectroscopy ${ }^{18)}$ or Raman spectroscopy ${ }^{19)}$ and the sheet resistance of stacked GO films. However, by these instruments, the obtained information is spatially 
averaged and the local electrical property can not be measured directly. Scanning probe microscopy proved to be useful for studying local properties. As the micro/nano-scale electrical measurement, conductive atomic force microscopy (C-AFM) has been developed to evaluate the electrical properties of semiconductor materials ${ }^{20)}$ or carbon nanomaterials such as carbon nanotube ${ }^{21,22)}$ and reduced graphene oxide ${ }^{23) 24)}$. In this method, local electrical conductivity can be evaluated by mapping the local current with the voltage applied AFM cantilever.

Here, we apply the C-AFM measurement to EGO sheets for the observation of its electrical property. Additionally, associated with the analysis by XPS and $\mu$ RS, the relationship between EGO structure and the electrical property is discussed.

\section{Experimental methods}

\subsection{Sample preparation}

Electrochemically-exfoliated GO was synthesized with reference to Cao's report, that is two-step electrochemical exfoliation process $^{14)}$. Figure 1(a) shows the schematic image of electrochemical apparatus. Galvanostatic charging was applied to graphite foil $(99.8 \%$, Alfa Aesar, $0.5 \mathrm{~mm}$ thickness) in a two-electrode cell with a platinum wire as cathode and concentrated $\mathrm{H}_{2} \mathrm{SO}_{4}(97 \%$, Nacalai Tesque) as the electrolyte. Charging current was kept at $100 \mathrm{~mA}$ for $20 \mathrm{~min}$. For the second electrochemical step, the graphite foil was removed in the first electrolyte and potentiostatic charging was applied to the foil in a two-electrode cell with a platinum wire as cathode and $0.1 \mathrm{M}\left(\mathrm{NH}_{4}\right)_{2} \mathrm{SO}_{4}(99 \%$, Fujifilm Wako Pure Chemical Corp.) aqueous solution as the electrolyte. Charging voltage was kept at $10 \mathrm{~V}$ for around $10 \mathrm{~min}$ to exfoliate completely. Then the exfoliated graphite flakes were sonicated for $5 \mathrm{~min}$ and centrifuged for $10 \mathrm{~min}$ to remove the impurity including $\left(\mathrm{NH}_{4}\right)_{2} \mathrm{SO}_{4}$. After purification, the dispersion was maintained for 24 hours to precipitate and remove any aggregations.

For comparison, we used the chemically oxidized GO (CGO), which was synthesized by modified Hummers method reported before ${ }^{25}$. Briefly, graphite powder (Ito Graphite Co., Ltd., Z-100, the average particle size:60 $\mu \mathrm{m})$ was mixed with $\mathrm{NaNO}_{3}(98 \%$, Nacalai Tesque), concentrated $\mathrm{H}_{2} \mathrm{SO}_{4}\left(97 \%\right.$, Nacalai Tesque) and $\mathrm{KMnO}_{4}(99 \%$, Nacalai Tesque) for 3 days in the ice bath. Then, $5 \% \mathrm{H}_{2} \mathrm{SO}_{4}$ was added to the liquid obtained and the mixture was stirred for 2 hours. Furthermore, $\mathrm{H}_{2} \mathrm{O}_{2}(30 \%$, Nacalai Tesque) was added to the mixture and stirred for 2 hours. The mixture was purified by exchanging the supernatant for ultrapure water after centrifuge. 


\subsection{Sample characterization}

Both prepared GO sheets were deposited on the highly doped p-type Si substrate with $300 \mathrm{~nm} \mathrm{SiO}_{2}$ by spin-coating for the chemical and structural analysis. The chemical condition and the structure of each GO sheet was characterized by X-ray photoelectron spectroscopy (XPS, JEOL JPS-9010TRX) and micro Raman spectroscopy ( $\mu$ RS, Lucir). In the XPS measurement, a Mg target was applied using an acceleration voltage and current of $10 \mathrm{kV}$ and $10 \mathrm{~mA}$, respectively. $\mu \mathrm{RS}$ observation was conducted with a $532 \mathrm{~nm}$ laser as the excitation source. AFM and C-AFM measurement were performed under ambient condition using MFP-3D (Oxford Instruments). Rh-coated Si cantilevers (Hitachi High-Tech, SI-DF3-R, spring const. $1.6 \mathrm{~N} \mathrm{~m}^{-1}$ ) were employed for current mapping in contact mode. Al-backside-coated Si cantilevers (Hitachi High-Tech, SI-DF40, spring const. $35 \mathrm{~N} \mathrm{~m}^{-1}$ ) were used for amplitude modulation (AM) AFM topographic imaging. During C-AFM measurement, a force of $35 \mathrm{nN}$ was maintained, and $-1 \mathrm{~V}$ was applied to the cantilever. For an electrode to connect the sheet, $\mathrm{Au} / \mathrm{Ti}$ film (100 $\mathrm{nm} \mathrm{Au}$ and $10 \mathrm{~nm} \mathrm{Ti})$ was deposited on a part of the GO-coated substrate as shown in Fig. 1(b).

\section{Results and discussion}

Figure 1(c) and 1(d) show the graphite products after each step of anodization. After first step of the anodization ( $100 \mathrm{~mA}$ for $20 \mathrm{~min}$ in concentrated $\left.\mathrm{H}_{2} \mathrm{SO}_{4}\right)$, the immersed part of graphite foil was expanded, which implies that sulfates were intercalated between layers of graphite. After the second step of anodization (10 V in $\left.0.1 \mathrm{M}\left(\mathrm{NH}_{4}\right)_{2} \mathrm{SO}_{4}\right)$, the graphite intercalated compounds were peeled into small flakes as shown in Fig. 1(d). Notably, the solution after electrochemical exfoliation was transparent. After the second step of the electrochemical treatment, the solution with flake-like products was sonicated. Figure 1(e) shows the solution after sonication and then kept for 1 days. Unlike just after electrochemical processes, the color of supernatant have changed into brown by the sonication. This color change suggested the existence of dispersed GO sheets. The EGO sheet deposited on the Si substrate was observed by AFM to confirm the number of layer and the size of EGO sheet, as shown in Fig. 2(a). The thickness was $1 \mathrm{~nm}$, which means that EGO sheet was mono-layer ${ }^{26)}$.

Several researchers have reported the one-step synthesis of electrochemicallyexfoliated GO in different conditions ${ }^{13,15,17)}$. In these graphite anodization process, the high positive voltage around $10 \mathrm{~V}$ is applied in the aqueous solution of sulfate to insert the 
anions into the graphite layers and to produce gases by water decomposition to oxygen simultaneously. In these method, the obtained GO sheets are usually multi-layer because graphite is exfoliated before sufficient sulfate intercalation. By the two-step anodization, sulfate was adequately inserted into graphite at the first step anodization in concentrated sulfuric acid without water decomposition, and these graphite intercalated compounds were further anodized in the aqueous solution of ammonium sulfate for the exfoliation. Remarkably, the graphite could not be separated into GO sheet immediately after anodization, as shown in Fig. 1(d), which means that the role of two-step anodization was broadening of the distance and weakening the interaction between each graphite layers, and the obtained graphite products were physically exfoliated by sonication.

The difference of chemical conditions and oxygen functional groups attached with each GO sheet was determined by the XPS analysis. Figures 3(a) and (b) show the XPS C 1s spectra of CGO and EGO, respectively. The spectra were calibrated to the Si $2 p$ peak at $103.5 \mathrm{eV}$ derived from Si-O bond. Furthermore, C 1s spectra were deconvoluted to 6 peaks, i.e., $\mathrm{sp}^{2} \mathrm{C}=\mathrm{C}$ at $284.4 \mathrm{eV}, \mathrm{sp}^{3} \mathrm{C}-\mathrm{C}$ at $285.0 \mathrm{eV}, \mathrm{C}-\mathrm{OH}$ at $286.2 \mathrm{eV}, \mathrm{C}-\mathrm{O}-\mathrm{C}$ at $286.9 \mathrm{eV}, \mathrm{C}=\mathrm{O}$ at $287.9 \mathrm{eV}$, and $\mathrm{COOH}$ at $289.1 \mathrm{eV}$ following the previous report ${ }^{24)}$. From the peak fitting result, the percentage of the chemical moieties $\left(P_{\mathrm{i}}\right)$ and the ratio of oxygen to carbon $\left(R_{\mathrm{O} / \mathrm{C}}\right)$ on GO sheets were shown in Table I. The $R_{\mathrm{O} / \mathrm{C}}$ was calculated by the equation below:

$$
R_{\mathrm{O} / \mathrm{C}}=\frac{P_{\mathrm{C}-\mathrm{OH}}+\frac{1}{2} P_{\mathrm{C}-\mathrm{O}-\mathrm{C}}+P_{\mathrm{C}=\mathrm{O}}+2 P_{\mathrm{COOH}}}{P_{\mathrm{C}=\mathrm{C}}+P_{\mathrm{C}-\mathrm{C}}+P_{\mathrm{C}-\mathrm{OH}}+P_{\mathrm{C}-\mathrm{O}-\mathrm{C}}+P_{\mathrm{C}=\mathrm{O}}+P_{\mathrm{COOH}}}
$$

There was little difference between the $R_{\mathrm{O} / \mathrm{C}}$ of ca. 0.40 for CGO and EGO. However, the main oxygen functional group on EGO was the hydroxyl group, whereas that on CGO was the epoxide group. This difference can be attributed to the oxidation and exfoliation process of each GO and may change the electrical property of each GO sheet.

Raman spectroscopy is one of the powerful tools to characterize the structure of graphene-like materials. The Raman spectra of both GO sheets were shown in Figs 4(a) and (b). In the spectra, there were two clear peaks derived from GO sheets, one was around $1350 \mathrm{~cm}^{-1}$ called D peak and the other was around $1600 \mathrm{~cm}^{-1}$ called G peak ${ }^{27)}$. Slight differences were found in these two spectra, such as the position of G peak and the intensity of D peak. At first, G peak of GO sheets is the combination of two peaks, that is, $G$ peak derived from the graphitic lattice around $1580 \mathrm{~cm}^{-1}$ and $D^{\prime}$ peak by the disorder-induced graphitic structure around $1610 \mathrm{~cm}^{-128)}$. In comparison with the CGO spectrum, the peak around $1600 \mathrm{~cm}^{-1}$ of EGO was red shifted due to the D' peak weakening, which means that EGO sheets had fewer defects than CGO. Next, D peak, 
which is derived from the breathing modes of six-atom rings, requires a defect for its activation. In the discussion about the structure of graphene-like materials by Raman spectra, the ratio of $\mathrm{D}$ peak and $\mathrm{G}$ peak intensity $\left(\mathrm{I}_{\mathrm{D}} / \mathrm{I}_{\mathrm{G}}\right)$ and the width of peaks are common parameters. Generally, when the defects at GO sheet are repaired, all of the peaks become sharp, the intensity of $\mathrm{G}$ peak increase and that of $\mathrm{D}$ peak decrease. However, it is reported that the intensity of $\mathrm{D}$ peak decreases when the defect density at GO sheets increases and the distance between defects decreases to less than $5 \mathrm{~nm}$. This is due to the breakdown of the six-atom rings lattice structure area, which is the origin of D peak ${ }^{29)}$. It is confirmed that EGO has sharper $\mathrm{D}$ peak and higher $\mathrm{I}_{\mathrm{D}} / \mathrm{I}_{\mathrm{G}}$ value than those of $\mathrm{CGO}$, which indicates that EGO had highly ordered structure.

Local electrical conductivity of EGO was measured by C-AFM in contact mode as shown in Fig. 5(a). EGO sheets were deposited on the thermally-grown $\mathrm{SiO}_{2}(300 \mathrm{~nm}$ thickness) surface, which is an insulator. After the $\mathrm{Au} / \mathrm{Ti}$ electrode deposition, we performed $\mathrm{C}$-AFM measurement at the boundary region of the $\mathrm{Au} / \mathrm{Ti}$ electrode in order to obtain the current mapping images of EGO sheet connected to the electrode. Therefore, the C-AFM configuration in this study can detect the current flowing through the EGO sheet laterally. The white region in Fig. 5(a) upper side, which showed the current more than 20 $\mu \mathrm{A}$, is the area of the deposited $\mathrm{Au} / \mathrm{Ti}$ electrode. The black region in Fig. 5(a), which detects less than $1 \mu \mathrm{A}$, can be the exposed $\mathrm{SiO}_{2}$, the EGO sheets without connection to $\mathrm{Au} / \mathrm{Ti}$ electrode, or the position in a connected EGO sheet with current below the detection limit. Most of the grey region in Fig. 5(a) are the EGO sheets connected to the electrode. In this region, the detected current gradually decreases as the distance from the electrode increases due to the integrated resistivity of the EGO sheet. The signal in an EGO sheet shows the variation at a certain distance from the electrode, and forms the domain-like part in the current mapping image. We considered that domain feature of current mapping reflects the inhomogeneous local conductivity of the EGO sheet. When the probe is placed on the low-conductive domain, the current flows through low-conductive domain at first, leading to the low-current signal. On the other hand, when the tip contacts with the high conductive domain, current flows only through the conductive path in the EGO sheet to the electrode, leading to the higher current signal. This difference results in the variation of the measured current in an EGO sheet, and the formation of high- and low-conductive domains in Fig. 5(a). Schematic images are shown in Figure S1 in the Supplementary Data. The low conductive domain derives from the EGO's structural feature. As shown in Fig. 3 and 4, EGO sheets have some oxygen functional groups and defects which were formed in the 
synthesis process, and those parts should have lower electrical conductivity than graphitic domain.

Figure 5(b) and 5(c) show the current profile and the corresponding resistance profile following by the Ohm's law. As shown in Fig. 5(c), the intercept value is $90 \mathrm{k} \Omega$, which means the contact resistance at EGO-electrode and EGO-probe contacts. In the previous report, the contact resistance between graphene and $\mathrm{Cr} / \mathrm{Au}$ electrode was measured by 2 and 4-probe measurement. The measured contact resistance ranged from $10^{3}$ to $10^{6} \Omega$ $\mu \mathrm{m}^{30)}$, which agrees with our experimental results. Furthermore, the measured current was inverse proportion to the distance from electrode to the measured point except for the low-conductive domains, which means that current detours in order to avoid the defects as shown in Fig. S2. These results indicate that the current measurement was not restricted by the contact resistance but reflected the resistance of EGO sheet. Regarding the triangle region on EGO sheet formed by the tip and the electrode side connected with EGO sheet as the electrical conduction path, the electrical conductivity can be calculated by utilizing the relation between resistance and distance ${ }^{31)}$. The resistance of the micro area surrounded by dashed line in Figure 5(d) is given by

$$
d R=\rho \frac{d x}{\left\{\frac{x}{L}\left(W_{\mathrm{e}}-W_{\mathrm{t}}\right)+W_{\mathrm{t}}\right\} T}
$$

where $\rho$ is the resistivity of the EGO sheet, $\mathrm{L}$ is the distance between the tip and the electrode, $T$ is the thickness of the sheet, $W_{\mathrm{e}}$ is the connected length between the sheet and the electrode, and $W_{\mathrm{t}}$ is the diameter of the tip contact area. The contact area of the tip and EGO sheet was estimated to be $13.3 \mathrm{~nm}^{2}$ by following the Hertzian contact mechanics model and $W_{\mathrm{t}}$ was evaluated to be $4 \mathrm{~nm}$. Then, the electrical conductivity $\sigma$ of the sheet was calculated by integrating the Eq. (2) and converting $\rho$ as $\sigma$, that is,

$$
\sigma=\frac{L}{R} \frac{\ln \left(\frac{W_{\mathrm{e}}}{W_{\mathrm{t}}}\right)}{T\left(W_{\mathrm{e}}-W_{\mathrm{t}}\right)}
$$

By using the experimental parameters of $W_{\mathrm{e}}$ as $1.7 \mu \mathrm{m}$ and $L / R$ as $0.13 \mathrm{M} \Omega \mu \mathrm{m}^{-1}$ which is approximate to the fitting result shown in Fig. 5(c) except for the low-conductive domains, the calculated electrical conductivity of the right side of EGO sheet was about $27000 \mathrm{~S} \mathrm{~m}^{-1}$, which was similar to that of reduced EGO membrane in Cao's report ${ }^{14}$. The resistance of EGO sheet was also confirmed by its I-V characteristic as shown in Fig. S3 in the Supplementary Data, which was calculated to be $250 \mathrm{k} \Omega$, almost the same as that estimated by the current mapping results. In the previous report, C-AFM measurement of reduced CGO demonstrated its electrical conductivity and the existence of low-conductive domains 
with the size of several tens to hundreds nanometers. The calculated electrical conductivity was $0.2 \mathrm{~S} \mathrm{~m}^{-126)}$. Despite some quantity of oxygen functional groups on EGO sheets, EGO was proved to be more conductive than reduced CGO.

From the chemical, structural characterization and the local electrical conductivity, the possible theory of the relationship between the structure and the electrical property of the GO sheet can be proposed. The main factor of EGO's high electrical conductivity is owing to the ordered graphitic structure. EGO sheets have fewer defects than $\mathrm{CGO}$, which was revealed by the Raman spectroscopy. Generally, the electrical path is the $\mathrm{sp}^{2}$ domains on the sheet, and this domain can be destroyed by the strong oxidant such as $\mathrm{KMnO}_{4}$ or nitric acid in the fabrication process of CGO. On the other hand, EGO was prepared by moderate oxidization of graphite and it was completely exfoliated by electrochemical process, which leads to the ordered structure. Additionally, the types of oxygen functional groups may affect the electrical conductivity of both GO sheets. From the XPS spectra, the species of oxygen functional groups on each GO was proved to be different although the $R_{\mathrm{O} / \mathrm{C}}$ was almost the same. The main oxygen functionality on CGO was epoxide which was connected to in-plane of the sheet according to the Lerf-Klinowski model ${ }^{32)}$, hence conductive path was separated. In contrast, the main oxygen containing group on EGO was hydroxyl group. Ghaderi have reported that it is stable for hydroxyl group to connect to the defect, disordered or edge parts of the sheet confirmed by density functional theory simulation $^{33)}$. Because hydroxyl group on EGO sheet do not conect to the graphitic domain which is the conduction path on the sheet, EGO sheet has higher electrical conductivity than CGO.

\section{Conclusions}

We investigated the electrical property of mono-layer EGO sheet by C-AFM. Without the post-treatment of reduction, the EGO sheet shows high conductivity about $27000 \mathrm{~S} \mathrm{~m}^{-1}$. The current mapping image of EGO sheets proved the existence of low-conductivity domains scattered on the EGO sheet which may be due to defects on the sheet. Combining C-AFM measurements with the chemical and structural analysis by XPS and $\mu$ RS, the high electrical conductivity of EGO was proved, even though there was little difference of XPS and $\mu \mathrm{RS}$ spectra between EGO sheet and CGO sheet. Although higher resolution images are necessary for further discussion about the atomic-scale structure and the electrical property of GO sheets, our report shows the potential for evaluating the electrical property of graphene-like material directly and the achieved results will be helpful for a range of 
electronics application.

\section{Acknowledgments}

This work was partially supported by JSPS KAKENHI grant number JP18K18946, the Iketani Science and Technology Foundation and the Murata Science Foundation.

\section{References}

1) K. S. Novoselov, A.K. Geim, S. V. Morozov, D. Jiang, Y. Zhang, S. V. Dubonos, I. V. Grigorieva, and A. A. Firsov, Nature 306, 666 (2004)

2) C. Lee, X. Wei, J.W. Kysar, and J. Hone, Science 321, 385 (2008)

3) A.A. Balandin, S. Ghosh, W. Bao, I. Calizo, D. Teweldebrhan, F. Miao, and C.N. Lau, Nano Lett. 8, 902 (2008)

4) Y. Shao, J. Wang, H. Wu, J. Liu, I.A. Aksay, and Y. Lin, Electroanalysis 22, 1027 (2010)

5) J.K. Wassei and R.B. Kaner, Mater. Today 13, 52 (2010)

6) C. Liu, Z. Yu, D. Neff, A. Zhamu, and B.Z. Jang, Nano Lett. 10, 4863 (2010)

7) X. Li, W. Cai, J. An, S. Kim, J. Nah, D. Yang, R. Piner, A. Velamakanni, I. Jung, E.

Tutuc, S.K. Banerjee, L. Colombo, and R.S. Ruoff, Science 324, 1312 (2009)

8) C. Berger, Z. Song, T. Li, X. Li, A.Y. Ogbazghi, R. Feng, Z. Dai, N. Alexei, M.E.H. Conrad, P.N. First, and W.A. De Heer, J. Phys. Chem. B 108, 19912 (2004)

9) S. Mao, H. Pu, and J. Chen, RSC Adv. 2, 2643 (2012)

10) J.W. Suk, A. Kitt, C.W. Magnuson, Y. Hao, S. Ahmed, J. An, A.K. Swan, B.B.

Goldberg, and R.S. Ruoff, ACS Nano 5, 6916 (2011)

11) J.I. Paredes, S.V. Rodil, A.M. Alonso, and J.M.D. Tascón, Langmuir 24, 10560 (2008)

12) S. Yang, S. Brüller, Z.S. Wu, Z. Liu, K. Parvez, R. Dong, F. Richard, P. Samorì, X.

Feng, and K. Müllen, J. Am. Chem. Soc. 137, 13927 (2015)

13) K. Parvez, Z.S. Wu, R. Li, X. Liu, R. Graf, X. Feng, and K. Müllen, J. Am. Chem. Soc. 136, 6083 (2014)

14) J. Cao, P. He, M.A. Mohammed, X. Zhao, R.J. Young, B. Derby, I.A. Kinloch, and R.A.W. Dryfe, J. Am. Chem. Soc. 139, 17446 (2017)

15) K. Parvez, R. Li, S.R. Puniredd, Y. Hernandez, F. Hinkel, S. Wang, X. Feng, and K. Müllen, ACS Nano 7, 3598 (2013)

16) J.M. Munuera, J.I. Paredes, M. Enterría, A. Pagán, S. Villar-Rodil, M.F.R. Pereira, J.I. Martins, J.L. Figueiredo, J.L. Cenis, A. Martínez-Alonso, and J.M.D. Tascón, ACS Appl. 
Mater. Interfaces 9, 24085 (2017)

17) J. Lu, J. Yang, J. Wang, A. Lim, S. Wang, and K.P. Loh, ACS Nano 3, 2367 (2009)

18) D. Yang, A. Velamakanni, G. Bozoklu, S. Park, M. Stoller, R.D. Piner, S. Stankovich,

I. Jung, D.A. Field, C.A. Ventrice, and R.S. Ruoff, Carbon 47, 145 (2009)

19) A.C. Ferrari and D.M. Basko, Nat. Technol. 8, 235 (2013)

20) C. Li, Y. Bando, and D. Golberg, ACS Nano 4, 2422 (2010)

21) H. Dai, E.W. Wong, and C.M. Lieber, Science 272, 523 (1996)

22) Y. Otsuka, Y. Naitoh, T. Matsumoto, and T. Kawai, Appl. Phys. Lett. 82, 1944 (2003)

23) J.M. Mativetsky, E. Treossi, E. Orgiu, M. Melucci, G.P. Veronese, P. Samorì, and V. Palermo, J. Am. Chem. Soc. 132, 14130 (2010)

24) Y. Tu, H. Nakamoto, T. Ichii, T. Utsunomiya, O.P. Khatri, and H. Sugimura, Carbon 119, 82 (2017)

25) M. Hirata, T. Gotou, S. Horiuchi, M. Fujiwara, and M. Ohba, Carbon 42, 2929 (2004)

26) Y. Tu, T. Ichii, T. Utsunomiya, and H. Sugimura, Appl. Phys. Lett. 106, 133105 (2015)

27) K.N. Kudin, B. Ozbas, H.C. Schniepp, R.K. Prud'homme, I.A. Aksay, and R. Car, Nano Lett. 8, 36 (2008)

28) R.J. Nemanich and S.A. Solin, Phys. Rev. B 2, 392 (1979)

29) M.M. Lucchese, F. Stavale, E.H.M. Ferreira, C. Vilani, M.V.O. Moutinho, R.B. Capaz, C.A. Achete, and A. Jorio, Carbon 48, 1592 (2010)

30) K. Nagashio, T. Nishimura, K. Kita, and A. Toriumi, Jpn. J. Appl. Phys. 49, 0513041 (2010)

31) Y. Tu, T. Utsunomiya, T. Ichii, and H. Sugimura, J. Vac. Sci. Technol. B 35, 03D110 (2017)

32) H. He, T. Riedl, A. Lerf, and J. Klinowski, J. Phys. Chem. 100, 19954 (1996)

33) N. Ghaderi and M. Peressi, J. Phys. Chem. C 114, 21625 (2010) 


\section{Figure Captions}

(a)

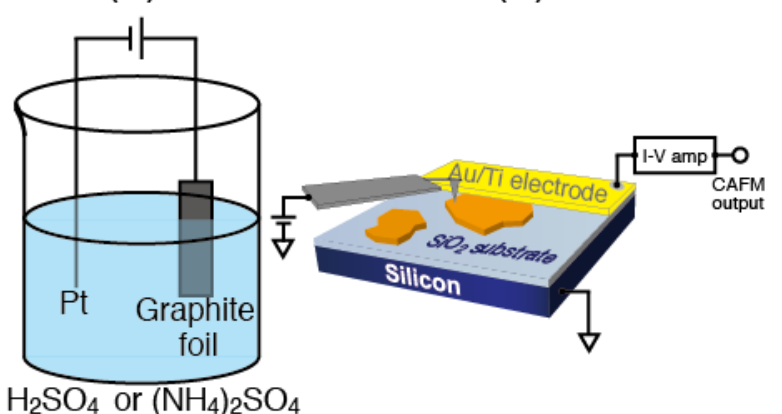

(c)

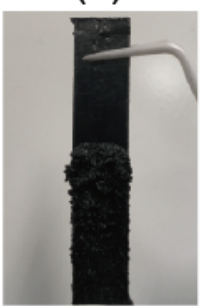

(d)

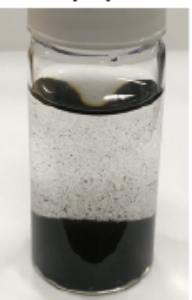

(e)

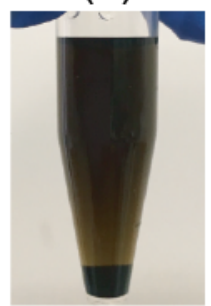

Figure 1 Schematic images of (a) electrochemical exfoliation of graphite and (b) the C-AFM measurement. The photographs of graphite products (c) after first anodization (100 $\mathrm{mA}$ for 20 min in concentrated $\mathrm{H}_{2} \mathrm{SO}_{4}$ ), (d) after second anodization ( $10 \mathrm{~V}$ for $10 \mathrm{~min}$ in $\left.0.1 \mathrm{M}\left(\mathrm{NH}_{4}\right)_{2} \mathrm{SO}_{4}\right)$, and (e) after sonication. 
(a)

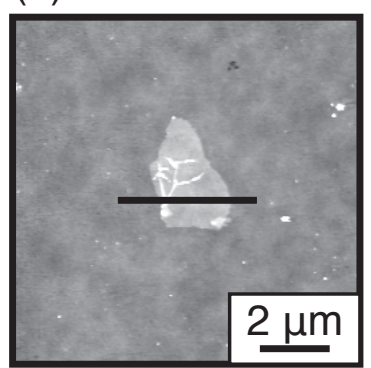

(b)

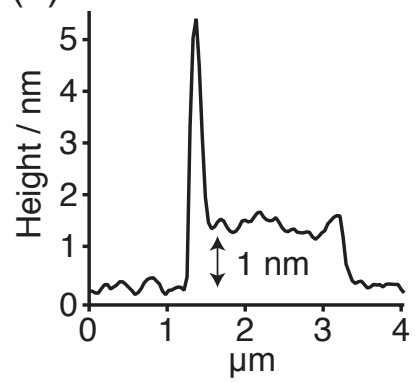

Figure 2 (a) AFM topographic image and (b) the cross-sectional profile along the line of the EGO sheet on the $\mathrm{SiO}_{2} / \mathrm{Si}$ substrate. 


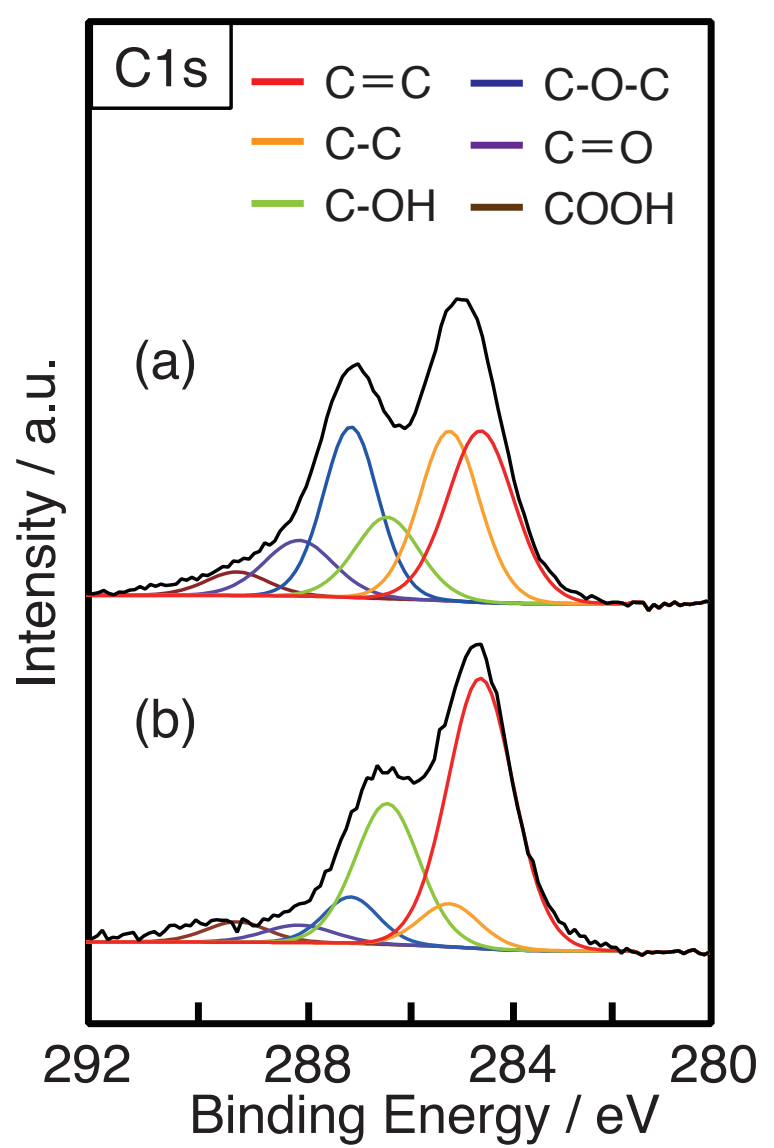

Figure 3 XPS C 1s spectra of GO synthesized by (a) modified Hummers' method (CGO) and (b) electrochemical exfoliation (EGO). The spectra were calibrated to the Si 2p peak at $103.5 \mathrm{eV}$. Each spectrum was deconvoluted to six peaks as shown in the figure. 
Table I The percentage of the chemical species and the ratio of oxygen to carbon in each GO calculated by peak areas of deconvoluted XPS C 1s spectra.

\begin{tabular}{|c|c|c|c|c|c|c|c|}
\hline & \multicolumn{6}{|c|}{ Peak Percentage / \% } & \\
\hline & $P_{\mathrm{C}=\mathrm{C}}$ & $P_{\mathrm{C}-\mathrm{C}}$ & $P_{\mathrm{C}-\mathrm{OH}}$ & $P_{\mathrm{C}-\mathrm{O}-\mathrm{C}}$ & $P_{\mathrm{C}=\mathrm{O}}$ & $P_{\mathrm{COOH}}$ & $R_{\mathrm{O} / \mathrm{C}}$ \\
\hline $\mathrm{CGO}$ & 27.4 & 24.6 & 12.7 & 22.5 & 9.3 & 3.5 & 0.40 \\
\hline EGO & 51.7 & 7.4 & 26.3 & 7.4 & 3.5 & 3.7 & 0.41 \\
\hline
\end{tabular}




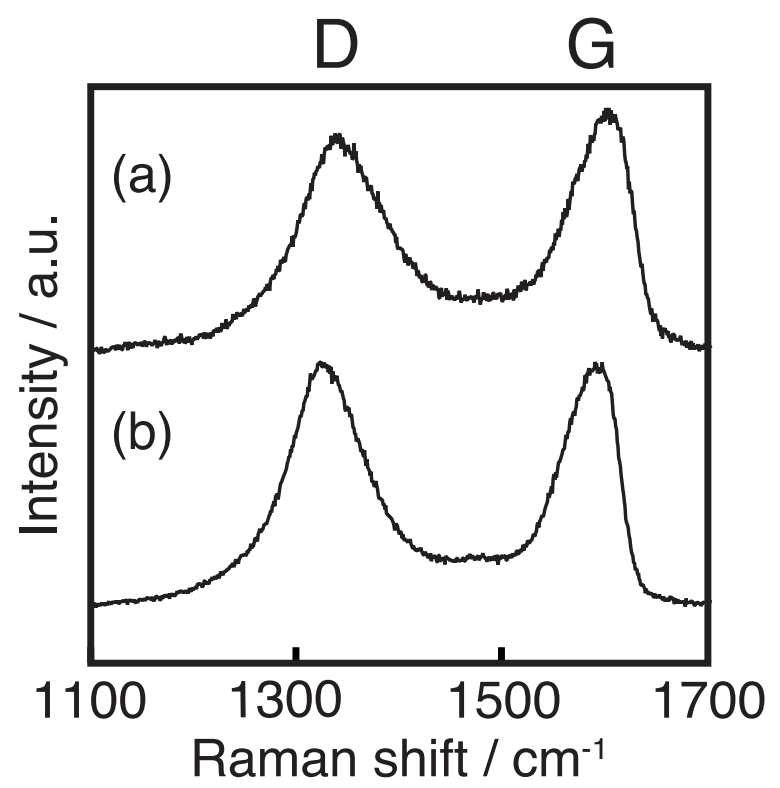

Figure $4 \mu \mathrm{RS}$ spectra of GO synthesized by (a) modified Hummers' method (CGO) and (b) electrochemical exfoliation (EGO). 
(a)
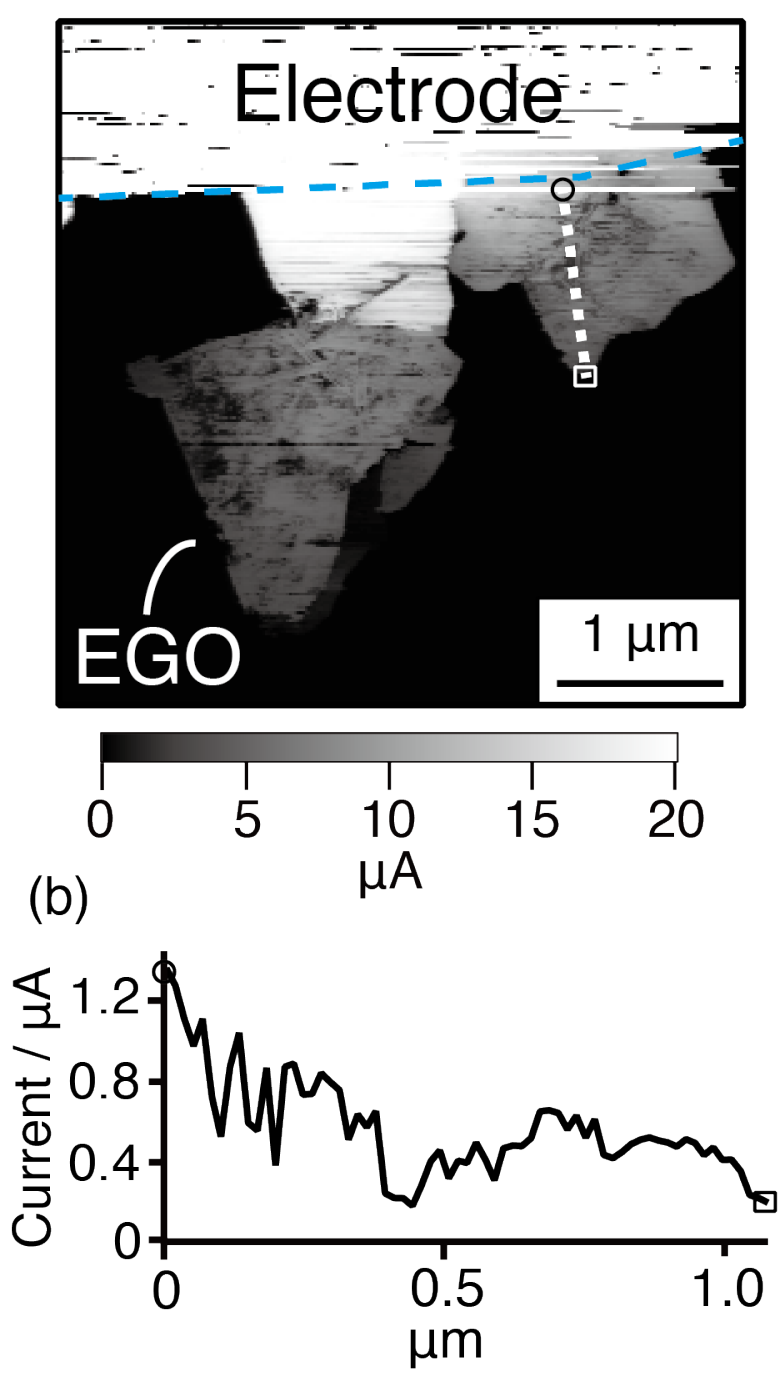

(c)

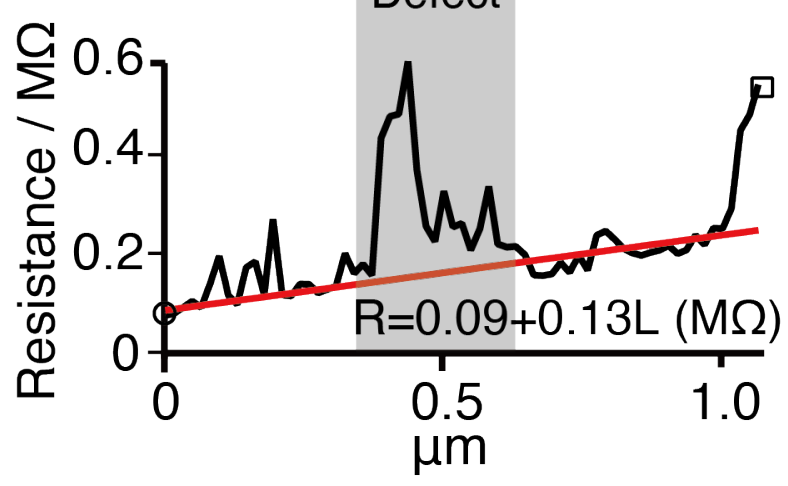




\section{(d) Au electrode}

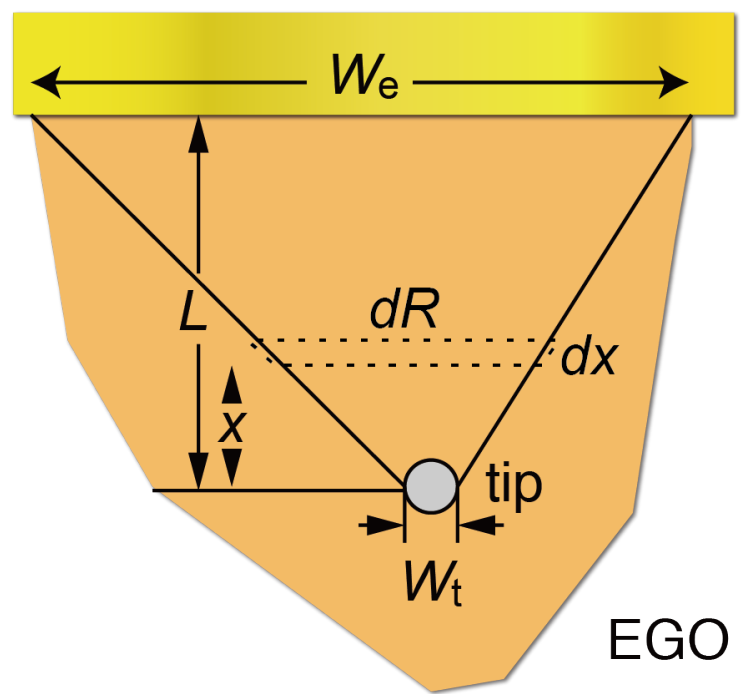

Figure 5 (a) Local current mapping of GO sheets synthesized by electrochemical method connected to the $\mathrm{Au} / \mathrm{Ti}$ electrode with the tip voltage of $-1 \mathrm{~V}$. (b) The current and (c) the corresponding resistance profile along the line. (d) A schematic image of C-AFM measurement for estimating the electrical conductivity of an EGO sheet. 